\title{
Intervenção antibullying no contexto escolar: Estudo de viabilidade ${ }^{1}$
}

\author{
Anti-bullying intervention in school context: Feasibility study \\ Intervención anti-bullying en el contexto escolar: Estudio de viabilidad
}

Recebido: 28/06/2021 | Revisado: 06/07/2021 | Aceito: 09/07/2021 | Publicado: 18/07/2021

\author{
Grazielli Fernandes \\ ORCID: https://orcid.org/0000-0003-3545-403X \\ Universidade La Salle, Brasil \\ E-mail: graziellifernandes@gmail.com \\ Débora Dalbosco Dell'Aglio \\ ORCID: https://orcid.org/0000-0003-0149-6450 \\ Universidade La Salle, Brasil \\ E-mail: dddellaglio@gmail.com
}

\begin{abstract}
Resumo
O objetivo deste estudo foi avaliar a viabilidade de uma intervenção antibullying no contexto escolar, a partir de critérios quantitativos e qualitativos. A intervenção grupal, realizada em oito encontros semanais, foi aplicada a nove adolescentes dos anos finais do Ensino Fundamental de uma escola pública do Brasil. Os instrumentos utilizados foram: Medida de Avaliação da intervenção, Ficha de Avaliação do Observador e Diário de campo. Os aspectos quantitativos observados foram: adesão dos participantes à intervenção; habilidades sociais e integridade/fidelidade do moderador; satisfação, clareza/compreensão e aplicação dos conteúdos na vida diária. A Análise de Conteúdo do tipo temática identificou três temas: Aplicação das aprendizagens, Engajamento e protagonismo e Avaliação da intervenção. Os resultados demonstraram uma avaliação positiva da intervenção e da moderadora, com engajamento dos participantes nas atividades propostas. A partir da avaliação, foram propostas alterações em algumas atividades da intervenção e alteração em um item da Ficha de Avaliação. Pode-se concluir que a proposta da intervenção tem potencial e é viável para estudos futuros.
\end{abstract}

Palavras-chave: Bullying; Intervenção; Estudo de viabilidade; Adolescentes.

\begin{abstract}
The aime of this study was to evaluate the feasibility of an anti-bullying intervention in the school context, from quantitative and qualitative criteria. The group intervention, carried out in eight weekly meetings, was applied to nine Elementary School adolescents from a public school in Brazil. The instruments used were: Intervention Evaluation Measure, Observer Evaluation Form and Field Diary. The quantitative aspects observed were: participants' adherence in the intervention; moderator's social skills and integrity/fidelity; satisfaction, clarity/understanding and application of contents in daily life. Thematic Content Analysis identified three themes: Application of learning, Engagement and protagonism and Evaluation of the intervention. The results demonstrated a positive evaluation of the intervention and the moderator, with participants' engagement in the proposed activities. Based on the analysis, changes were proposed in some activities of the intervention and in an item of the Evaluation Form. It can be concluded that the intervention proposal has potential and is viable for future studies.
\end{abstract}

Keywords: Bullying; Intervention; Feasibility study; Adolescents.

\section{Resumen}

El objetivo de este estudio fue evaluar la viabilidad de una intervención anti-bullying en el contexto escolar, con base en criterios cuantitativos y cualitativos. La intervención grupal, realizada en ocho encuentros semanales, se aplicó a nueve adolescentes de la educación primaria de una escuela pública en Brasil. Las herramientas utilizadas fueron: Medida de Evaluación de la Intervención, Ficha de Evaluación del Observador y Diario de Campo. Los aspectos cuantitativos observados fueron: adherencia de los participantes a la intervención; habilidades sociales e integridad / fidelidad del moderador; satisfacción, claridad / comprensión y aplicación de contenidos en la vida diaria. El análisis de contenido temático identificó tres temas: aplicación del aprendizaje, participación y protagonismo, y evaluación de la intervención. Los resultados mostraron una valoración positiva de la intervención y del moderador, con el compromiso de los participantes en las actividades propuestas. Desde los análisis se propusieron cambios en algunas actividades de la intervención y en un ítem de la Ficha de Evaluación. Se puede concluir que la propuesta de intervención tiene potencial y es viable para estudios futuros.

Palabras clave: Bullying; Intervención; Estudio de viabilidade; Adolescentes. 


\section{Introdução}

A violência juvenil é um problema global de saúde pública, incluindo, nesse contexto, práticas de bullying (Organização das Nações Unidas [ONU], 2014). Em relação ao conceito de bullying, Smith, Görzig e Robinson (2018) destacam que ainda há discussões no meio científico e, por isso, não existe um consenso entre os pesquisadores. Ainda assim, esta é a definição aceita atualmente: bullying é um processo que ocorre quando uma pessoa é vitimizada ou agredida de forma intencional por parte de um ou mais agressores, com desequilíbrio de poder entre agressor(es) e vítima(s) (Olweus, 1993; Olweus \& Limber, 2010). Os envolvidos em bullying podem desempenhar os papéis de vítimas, aqueles que sofrem bullying; agressores, aqueles que praticam bullying; agressores-vítimas, aqueles que praticam e sofrem bullying; e observadores (Olweus, 1993), que podem ser de quatro tipos: reforçadores das atitudes dos autores, assistentes dos autores, defensores das vítimas ou testemunhas silenciosas (Salmivalli, 2010).

O bullying pode ser praticado de forma direta ou indireta. A forma direta inclui bullying físico (bater, socar, chutar ou danificar pertences) e verbal (provocar, insultar, ameaçar). Entre as formas indiretas, estão a exclusão social, a prática de espalhar rumores e fofocas (Smith, 2014). Em relação ao cyberbullying, Ferreira e Deslandes (2018), em um estudo de revisão, concluem que este termo possui diferentes conceituações, mas há argumentos que defendem sua diferenciação em relação ao bullying. Nesse mesmo caminho, para Smith et al. (2018), o cyberbullying, tipo de violência praticado virtualmente (Smith, 2014), tem suas próprias características, e os critérios definidores de repetição e desequilíbrio de poder são mais complexos.

Um estudo com 40 países em desenvolvimento confirmou que uma média de $42 \%$ dos meninos e $37 \%$ das meninas foram expostos ao bullying (ONU, 2014). No Brasil, estudo utilizando dados da Pesquisa Nacional de Saúde Escolar (PeNSE, 2015), com 102.301 estudantes de todas as regiões do país, concluiu que $7,2 \%$ dos estudantes foram vítimas e $20,8 \%$, autores de bullying (Malta et al., 2014). Resultados recentes de pesquisa realizada com 764.518 estudantes de 37 países da Europa e América do Norte, em um ciclo de quatro anos (2002, 2006, 2010 e 2014), demonstram que a prevalência de cyberbullying foi sistematicamente menor que o bullying tradicional. No geral, em todos os países, 45,8\% dos que relataram cybervitimização também relataram bullying tradicional (46,5\% para meninos e 45,3\% para meninas), com amplas variações nos países (Cosma et al., 2020).

Em relação às consequências, as vítimas podem apresentar sintomas psicossomáticos $(\mathrm{Li}$, Sidibe, Shen, \& Hesketh, 2019); tristeza, solidão e insônia (Fleming \& Jacobsen, 2010); ideações e tentativas suicidas (Arango, Opperman, Gipson, \& Kin, 2016). Os autores também podem apresentar sintomas psicossomáticos, ansiedade e problemas de conduta (AlboresGallo, Sauceda-García, \& Roque-Santiago, 2011) e têm maior tendência para comportamentos de risco (Fleming \& Jacobsen, 2010; Mello et al., 2017). Devido aos problemas físicos e emocionais causados pelo bullying, diferentes programas de intervenção têm sido desenvolvidos. O estopim aconteceu na década de 1980, na Noruega, quando jovens desse país cometeram suicídio motivados por situações de bullying (Olweus \& Limber, 2010). Após esse fato, Dan Olweus (1993), por solicitação do Ministro da Educação Norueguês, implementou o primeiro programa de intervenção que se tem conhecimento, denominado Olweus Bullying Prevention Program (OBPP). Outros países, como Brasil (Silva et al., 2018a), Finlândia (Juvonen, Schacter, Sainio, \& Salmivalli, 2016), Itália (Palladino, Nocentini, \& Menesini, 2016), Estados Unidos (Menard \& Grotpeter, 2014), entre outros, já desenvolveram programas antibullying ou adaptaram ao seu contexto cultural o OBPP (caso dos Estados Unidos, por exemplo).

Na tentativa de reduzir os índices de bullying, pesquisadores têm desenvolvido programas com foco na prevenção; em ações direcionadas a aspectos que interferem nas relações entre os pares, como melhoria do clima escolar; em ações reativas, quando são adotadas atitudes após as situações de bullying; ou ainda em ações relacionadas ao apoio entre pares que apresentam aspectos reativos e proativos (Smith, 2011). Quanto aos modelos teóricos, há programas baseados em práticas 
restaurativas (Bonell et al., 2018), no modelo socioecológico (Rana et al., 2018), no modelo cognitivo-comportamental (Silva et al., 2016), na teoria da personalidade (Calvete et al., 2019), entre outros.

Estudos apontam que alguns componentes são imprescindíveis para o delineamento de intervenções, como abordagens com foco na empatia, rede de apoio, engajamento escolar e protagonismo juvenil. Nas dinâmicas de bullying, autores e testemunhas que encorajam as agressões ou atuam como assistentes não desenvolveram total ou parcialmente habilidades relacionadas à empatia (Olweus, 1993; Salmivalli, 2010). Por isso, atividades que envolvem empatia podem tornar agressores e observadores mais sensíveis e solidários em relação ao estado emocional de seus pares vitimizados (Lopez, 2009). Ao mesmo tempo, a rede de apoio surge como um elemento importante a ser trabalhado, já que estimula os participantes a olharem para seu contexto e perceberem quem são as pessoas significativas com as quais podem contar (Brito \& Koller, 1999), especialmente em situações de risco. Estudos revelam que uma rede de apoio sólida tem o potencial de proteger as pessoas de situações de risco, como bullying (Brito \& Koller, 1999), e que uma única amizade solidária entre pares é também um mecanismo de proteção a crianças e adolescentes (Harmelen et al., 2017).

Com o engajamento escolar, busca-se estimular o envolvimento dos estudantes com a escola como um todo (Lewis $e t$ al., 2011), de forma que percebam esse espaço como um ambiente protetivo e acolhedor, especialmente em situações que envolvam a violência entre os pares. Além de contribuir para a redução do envolvimento em bullying (Forster et al., 2019), o engajamento apresenta outros benefícios, como valorização e identificação dos estudantes com a instituição, melhores resultados acadêmicos e prevenção do abandono escolar (Fredricks, Blumenfeld, \& Paris, 2004). Diretamente relacionado ao engajamento escolar, o protagonismo juvenil é considerado uma forma de encorajar a participação ativa, construtiva e solidária dos adolescentes com foco na solução de problemas de sua realidade, como o bullying (Costa, 2000; Vivaldi, 2020). Alguns programas são delineados a partir da visão dos adolescentes como protagonistas na prevenção do bullying, pois se trata de um problema que os afeta diretamente, e são os pares que conhecem melhor os problemas de seus colegas (Tognetta, Souza, \& Lapa, 2019; Vivaldi, 2020). Pesquisas apresentam evidências de que essa participação ativa traz muitos benefícios para crianças e adolescentes, como o desenvolvimento da autoestima, do senso de identidade, da autoconfiança, da visão do futuro, da autodeterminação e da busca de plenitude humana por jovens (Costa, 2000).

Entretanto, apesar dos esforços de pesquisadores em desenvolver programas eficazes a determinadas comunidades escolares, as intervenções têm apresentado resultados modestos, o que requer entendimento dos motivos pelos quais há variação de eficácia (Smith, 2011). Por isso, é necessário ampliar as possibilidades teóricas e metodológicas para desenvolver propostas de intervenções que sejam avaliadas a partir de critérios sistemáticos específicos (Lopes, Silva, Castro, Bógus, \& Fracolli, 2013). O primeiro passo para a avaliação de uma intervenção é a condução do estudo de viabilidade (ou estudo piloto), realizado conforme critérios científicos indicados na literatura (National Institute for Health Research [NIHR], 2017). Dessa forma, neste estudo, tem-se por objetivo avaliar a viabilidade de uma intervenção ao bullying no contexto escolar, denominada \#NoBullying, com vistas a conscientizar e contribuir para a prevenção ao bullying, de acordo com a Lei de Combate ao Bullying (Lei 13.185/15).

\section{Descrição da Intervenção: \#NoBullying}

A intervenção \#NoBullying foi embasada em três perspectivas teóricas: Psicologia Positiva (Seligman, 2002), Método Experiencial (Martín-Quintana et al., 2009) e Metodologias Participativas (Santana \& Avanzo, 2014). O movimento da Psicologia Positiva considera variáveis associadas à experiência subjetiva positiva (emoções positivas, valores, otimismo, etc.); traços ou características individuais positivas (empatia, gratidão, perdão, etc.); e instituições positivas que visam a promover o potencial humano, tais como família, escolas, centros comunitários, etc., como fatores protetivos para um estado ótimo de funcionamento psicológico (Seligman, Steen, Park, \& Peterson, 2005). Assim, as atividades enfocam os aspectos positivos dos 
participantes para a mudança de comportamentos, com a valorização de suas experiências e vivências (Seligman, 2002).

Com o Método Experiencial, realiza-se um processo de reconstrução do conhecimento diário, estratégia que oferece aos participantes momentos de reflexão sobre suas próprias ideias, sentimentos e ações cotidianas (Martín-Quintana et al., 2009). Assim, propõe-se que os adolescentes sejam participantes ativos em todas as etapas da intervenção, e não meros receptores de conceitos e conhecimentos, proporcionando-se espaços para reflexão e tomada de consciência sobre seus sentimentos e atitudes no decorrer do processo. As Metodologias Participativas pressupõem que o adulto-pesquisador é responsável por delimitar o tema de investigação e por oferecer propostas que facilitem a participação de todos, mas são os próprios adolescentes os agentes de mudança em seus contextos de desenvolvimento, como a escola ou a comunidade em que vivem (Santana \& Avanzo, 2014).

A intervenção é composta por oito encontros grupais semanais, duração de dois meses, com a participação de um moderador (aplicador da intervenção) e de um observador, com média de duas horas por sessão, totalizando 16 horas. O número e a duração média dos encontros corresponde a outras intervenções e programas já implementados em diferentes países (Silva et al., 2018b). Foram definidas as temáticas de cada encontro, considerando os aspectos positivos, engajamento e protagonismo dos participantes no ambiente escolar. Com o objetivo de atingir de forma mais ampla a comunidade escolar, também foram elaboradas atividades que integram as famílias dos participantes e crianças de outras turmas da escola.

Para cada encontro, adotou-se a seguinte metodologia em comum: apresentação dos objetivos, retomada do conteúdo do encontro anterior, análise do tema no contexto familiar, aplicação de atividades relacionadas aos conteúdos, e uma reflexão final. Também foi proposto que os participantes desenvolvessem e aplicassem atividades sobre bullying e convivência escolar com crianças de turmas dos anos iniciais da própria escola. As atividades foram elaboradas no encontro 6 e aplicadas no encontro 7. No primeiro e último encontros, foram aplicados os instrumentos de avaliação (T1 e T2). Além das atividades comuns, em cada encontro foi abordado um tema e atividades específicas, como descrito na Tabela 1.

Tabela 1. Resumo dos encontros com objetivos e atividades.

\begin{tabular}{l} 
Encontro 1 - Apresentação \\
Objetivo: Apresentar a moderadora, o observador e o grupo participante. \\
Atividades \\
- “Quem sou eu?": apresentação dos participantes do grupo. \\
- Apresentação da intervenção: estrutura, duração e atividades. \\
\hline
\end{tabular}

Encontro 2 - Bullying

Objetivo: Possibilitar a compreensão sobre o fenômeno bullying.

Atividades

- Vídeo sobre bullying.

- Apresentação do tema bullying e reflexões com o grupo.

- Dramatização sobre bullying elaborada e encenada pelo grupo.

\section{Encontro 3 - Clima escolar}

Objetivo: Refletir sobre o clima escolar e atitudes que podem tornar a escola um lugar de convivência positiva e saudável.

Atividades

- Observação coletiva do clima escolar durante o intervalo do recreio.

- Jogo de tabuleiro antibullying com situações reais do ambiente escolar.

- Reflexões sobre a observação do clima escolar e as situações do Jogo, discussão sobre estratégias para melhorar o ambiente. 
Encontro 4 - Empatia e respeito às diferenças

Objetivo: Possibilitar a reflexão sobre a importância do respeito às diferenças e da empatia.

Atividades

- Atividade grupal sobre empatia.

- Jogo da memória sobre empatia e respeito às diferenças.

- Criação de atividade lúdica ou elaboração de cartaz com base no jogo.

- Vídeo sobre empatia e reflexões.

\section{Encontro 5 - Rede de apoio}

Objetivo: Possibilitar análise e reflexão sobre a rede de apoio.

Atividades

- Atividade grupal: Teia da amizade.

- Reflexões sobre Rede de Apoio (slides).

- “Quem faz parte da minha rede?”: produção e apresentação da rede de apoio.

Encontro 6 - Engajamento escolar

Objetivo: Promover o engajamento escolar, por meio da organização das atividades a serem desenvolvidas com alunos dos anos iniciais.

Atividades

- Produção coletiva de atividades para serem aplicadas.

- Organização do material e ensaio da aplicação das atividades.

\section{Encontro 7 - Protagonismo juvenil}

Objetivo: Tornar os participantes protagonistas na prevenção ao bullying no ambiente escolar, por meio de atividades por eles ministradas.

Atividades

- Ensaio das atividades a serem realizadas com os estudantes de $3^{\circ}$ ano.

- Realização das atividades pelos próprios participantes.

\section{Encontro 8 - Avaliação das aprendizagens positivas}

Objetivo: Finalizar a intervenção e aplicar os instrumentos pós-teste.

Avaliação final e confraternização.

Fonte: Autoras (2020).

\section{Metodologia}

Trata-se de um estudo de viabilidade destinado a construir a base para o estudo principal (Tickle-Degnen, 2013). Nesta etapa, o objetivo foi analisar o potencial de sucesso da intervenção e reduzir as ameaças à validade do estudo principal (NIHR, 2017; Tickle-Degnen, 2013). Com o estudo de viabilidade, pretende-se responder à questão: "Este estudo pode ser realizado?". Em um estudo de viabilidade, listam-se parâmetros incertos e descrevem-se métodos para melhorar sua precisão, para que o estudo principal tenha mais potencial de sucesso (NIHR, 2017).

Os critérios avaliados neste estudo foram: adesão (participação nas atividades propostas e presença ao longo da intervenção), avaliação da moderadora pelo observador (habilidades sociais e integridade/fidelidade para com os processos de implementação da intervenção), avaliação da intervenção pelos participantes (satisfação, clareza/compreensão e aplicação dos conteúdos na vida diária) (American Psychological Association [APA], 2002).

Para avaliação dos critérios qualitativos, dados foram obtidos por meio de: 1) um observador presente em cada encontro que preencheu a Ficha de Avaliação do Observador; 2) avaliação dos participantes por meio da Medida de Avaliação da Intervenção; e 3) Diário de Campo da moderadora. A triangulação no processo de coleta dos dados foi introduzida no delineamento para assegurar critérios de validade em pesquisa qualitativa (Lopes et al., 2013). Três observadores foram 
devidamente treinados para a tarefa, os quais se revezaram durante os encontros, de forma que sempre um deles estivesse presente.

Participaram deste estudo nove adolescentes do sexo feminino, com idades entre 13 e 17 anos $(m=13,77 ; d p=1,36)$, estudantes do $8^{\circ}$ e $9^{\circ}$ ano do Ensino Fundamental de escola pública do Sul do Brasil, localizada em um contexto de vulnerabilidade social. Os instrumentos utilizados foram:

- Medida de Avaliação da Intervenção (T2 - pós-intervenção): questionário com 11 questões objetivas que avalia a satisfação dos participantes com os encontros, clareza e compreensão dos conteúdos e a aplicação do aprendizado após o término da intervenção. Também inclui duas questões descritivas. O instrumento foi adaptado a partir do estudo de Durgante (2019).

- Ficha de Avaliação do Observador: questionário preenchido ao término dos encontros pelo observador previamente treinado, com vistas a avaliar os encontros e a performance da moderadora. São avaliadas as Habilidades Sociais do moderador em relação aos participantes (empatia, cordialidade, acolhimento, escuta das demandas) e a Integridade/Fidelidade do moderador quanto aos temas trabalhados em cada encontro. A Ficha foi adaptada a partir do estudo de Durgante (2019).

- Diário de Campo da moderadora: registros de observações, interpretações e inferências relativas a cada encontro da intervenção. Os dados obtidos dão suporte qualitativo às avaliações dos observadores, como critério adicional para maior qualidade metodológica (Creswell, 2003).

Realizou-se a avaliação de processo, com análise quantitativa dos dados coletados na Ficha de Avaliação do Observador e na Medida de Avaliação da Intervenção, utilizando-se o software IBM Statistical Package for the Social Sciences (SPSS Statistics, 22.0), para cálculo de médias e desvios-padrão. As respostas das questões descritivas e do Diário de Campo foram analisadas a partir da Análise de Conteúdo do tipo temática (Saldaña, 2009).

O projeto foi aprovado pelo Comitê de Ética em Pesquisa da Universidade La Salle. Após a concordância da escola, e foram convidados para participarem da intervenção alunos do oitavo e nono ano do Ensino Fundamental, pertencentes ao turno da manhã. Os pais/responsáveis dos alunos interessados assinaram o Termo de Consentimento Livre e Esclarecido (TCLE), e os adolescentes assinaram o Termo de Assentimento (TALE). Foram adotados os mesmos procedimentos de assinatura do TCLE e TALE em relação aos alunos dos anos iniciais que participaram das atividades do encontro 7.

\section{Resultados}

A partir da análise quantitativa dos dados, avaliou-se o critério de adesão, sendo que, dos 15 participantes selecionados para o estudo de viabilidade, nove concluíram a intervenção, todas estudantes do sexo feminino, havendo perda amostral de $40 \%$. Dos desistentes, as justificativas foram: inserção no mercado de trabalho e auxílio em casa com irmãos/sobrinhos mais novos ou com atividades domésticas (apenas um desistente não justificou sua saída).

$\mathrm{Na}$ Medida de Avaliação da Intervenção, os participantes responderam itens quanto a sua satisfação com a intervenção e com a moderadora, em escala de quatro pontos, preenchida no último encontro (T2), para os seguintes critérios: Satisfação com a intervenção (cinco itens), Clareza e compreensão dos conteúdos (dois itens) e Aplicação dos conteúdos na vida diária (quatro itens). A soma dos escores dos itens da Medida de Avaliação da Intervenção variou de 35 a 42 pontos, com

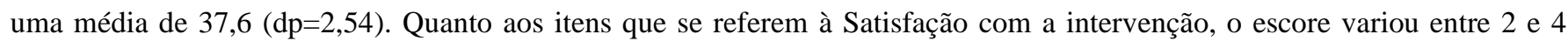
(média=3,71; dp=1,42), sendo os itens mais baixos os que se referem ao tempo (média=3,3) e horário (média=3,89) dos encontros. Quanto aos itens que se referem à Clareza e compreensão dos conteúdos e temas, o escore variou entre 3 e 4 (média $=3,27 ; \mathrm{dp}=0,88$ ), sendo o item mais baixo o que se refere ao entendimento do tema das sessões (média $=3,22$ ). Quanto aos itens que se referem à Aplicação dos conteúdos na vida diária, o escore variou entre 1 e 4 (média= 3,13; dp=1,50), sendo o item mais baixo o que se refere ao uso prático de alguma informação da intervenção (média $=3,00$ ). Desta forma, pode-se 
avaliar que os participantes mostraram-se satisfeitos com a intervenção e a moderadora.

$\mathrm{O}$ instrumento respondido a cada encontro pelos observadores procurou avaliar o cumprimento de itens relacionados às Habilidades Sociais da moderadora (cinco itens) e à Integridade/Fidelidade para com os processos de implementação da intervenção (sete itens), em uma escala de quatro pontos. Foi calculada a média dos escores atribuídos pelo observador nos oito encontros. A soma dos escores dos itens da Avaliação da moderadora variou entre 19 e 20, com uma média de 19,75 $(\mathrm{dp}=4,63)$. Os escores variaram entre três e quatro em cada item, com média de 3,95 para Habilidades Sociais e 3,96 para Integridade/Fidelidade. Os itens que não foram pontuados no valor máximo foram: "Verificou o humor dos participantes perguntando sobre como foi a semana, e se houve algum acontecimento significativo desde a última sessão" e "Usou diferentes estilos de comunicação verbal e não verbal para se fazer entender" (ambos com média 3,88). Quanto à Integridade/Fidelidade, os itens que não foram pontuados no valor máximo foram: "Realizou a condução das reflexões sobre o tema no contexto familiar" e "No final, retomou os temas abordados na sessão e fez encerramento" (ambos com média 3,88).

Nessa Ficha de Avaliação, preenchida pelos observadores presentes em cada encontro, percebeu-se a necessidade de suprimir um item ("Realizou a condução das reflexões sobre o tema no contexto familiar") nos encontros 1,7 e 8, nos quais essa tarefa não é mais solicitada. Dessa forma, concluiu-se que a Ficha não deve ser igual em todos encontros, sendo necessárias adaptações de acordo com o planejamento de cada encontro. Além disso, identificou-se a necessidade de treinamento dos observadores para o preenchimento da Ficha, destacando a importância de que sejam registradas observações sobre os itens que não foram cumpridos totalmente pela moderadora, além da pontuação atribuída, de forma que ela possa identificar em que ponto precisa melhorar. De forma geral, constatou-se que os instrumentos utilizados, tanto para os observadores como para os participantes, foram de fácil compreensão e preenchimento. No entanto, é necessária a retirada de um item da Ficha de Avaliação do Observador, que avalia a moderadora, para alguns dos encontros. Dessa forma, esse instrumento de avaliação precisa ser flexível, de acordo com as tarefas de cada encontro.

A análise temática (Saldaña, 2009) das respostas dos participantes nas questões descritivas, do conteúdo dos encontros gravados e transcritos e das anotações do Diário de Campo, indicou três temas: "Aplicação das aprendizagens", "Engajamento e protagonismo" e "Avaliação da intervenção".

No tema "Aplicação das aprendizagens", foram analisados os comentários e atitudes dos participantes quanto à aplicação prática do que foi abordado na intervenção, como atitudes de empatia e respeito às diferenças. Respostas que explicam o que as participantes aprenderam com a intervenção são, por exemplo: "nunca deixar alguém sozinho no recreio, sempre ajudar alguma pessoa e tentar compreender o que ela está passando" (Participante 1); "Em casa com minha família. Na escola quando alguém ou um amigo meu está mal” (Participante 2); “Quando vejo alguém sozinho eu tento o máximo pra essa pessoa ficar feliz e encontrar bastante amigos" (Participante 3); "Quando vi uma das minhas colegas sozinha e precisando de ajuda, fui e ajudei ela" (Participante 5). Durante os encontros, foi observado que as adolescentes adotaram atitudes de acolhimento e empatia com os próprios colegas. Um exemplo aconteceu no encontro 5, em que uma das meninas entrou na sala chorando, pois havia sido rejeitada por um menino. As demais participantes a acolheram com abraços e elogios, demonstrando empatia com a colega.

No tema "Engajamento e protagonismo", foi analisada a efetividade do processo de construção e replicação de atividades com as crianças de uma turma de terceiro ano da escola, nessas duas sessões. No primeiro encontro, as adolescentes foram informadas de que seriam responsáveis por desenvolver atividades com as crianças das turmas de $3^{\circ}$ ano sobre bullying e convivência escolar. A partir de dados do Diário de campo e das gravações, percebeu-se que as alunas aprovaram essa etapa da intervenção e demonstraram motivação e engajamento, tanto no momento da produção quanto no momento da condução das atividades com as crianças. Ao final do encontro 7, as meninas avaliaram as atividades: "A primeira atividade [que mantinha as crianças sentadas] eles não gostaram muito", e outra colega complementou: "Podemos fazer mais atividades para interagir 
mais com eles". As participantes também sugeriram que os próximos grupos da intervenção tenham mais tempo para os ensaios no encontro 6: "Acho que tem que ensaiar mais pra perder a vergonha".

No tema "Avaliação da intervenção", foram apresentadas as informações obtidas por meio das respostas abertas da Medida de Avaliação da Intervenção e das reflexões finais de cada encontro, registradas nas anotações dos observadores e no Diário de campo da pesquisadora. As participantes avaliaram a intervenção de forma positiva: "Foi tudo maravilhoso, eu amei demais" (Participante 3); "Para mim está tudo perfeito assim" (Participante 5); "Está tudo ótimo" (Participantes 6 e 8). Além disso, durante as reflexões finais de cada encontro, as adolescentes relataram que estavam gostando das atividades. Sempre após o término de cada encontro, a maioria das participantes permanecia na escola até o período final da tarde. Não sugeriram mudanças nas atividades grupais realizadas, mas, no encontro 6, ressaltaram a necessidade de mais tempo para o ensaio das atividades aplicadas com as crianças menores.

Quanto à estrutura da intervenção, a partir das análises do Diário de Campo da pesquisadora, dos registros dos observadores e das discussões conjuntas com a equipe, observaram-se aspectos que podem ser alterados. $\mathrm{O}$ primeiro aspecto observado foi sugerir novas composições dos grupos durante as atividades, para que os participantes possam interagir com outros colegas. No encontro 3, propõe-se que o jogo de tabuleiro seja a primeira atividade, como forma de apresentar sugestões para a elaboração do roteiro e posterior observação do ambiente escolar. No encontro 4, na atividade "Teia da Amizade", quando os participantes tiverem dificuldades em falar de si próprios, os colegas podem auxiliar. Na organização das atividades com as crianças, sugere-se que os participantes criem atividades mais interativas e tenham um maior tempo para o ensaio das atividades pelos adolescentes, de forma que se sintam mais seguros.

\section{Discussão}

Neste estudo, o objetivo foi avaliar a viabilidade da intervenção antibullying no contexto escolar, denominada \#NoBullying. Quanto ao critério de adesão, a taxa de $40 \%$ de desistência pode refletir características do grupo, uma vez que os participantes eram alunos de escola localizada em região de vulnerabilidade social. A intervenção ocorreu no turno inverso de aulas, e muitos dos desistentes informaram que assumiram outras tarefas no lar. A escola está localizada em uma região de baixa renda e, ainda que os adolescentes não exerçam atividades remuneradas, têm a responsabilidade de auxiliar em atividades domésticas. Conforme dados do Instituto Brasileiro de Geografia e Estatística (IBGE, 2015), meninos e meninas brasileiros, com percentuais de 37,6\% e 68,5\%, respectivamente, declararam que exercem tarefas domésticas, portanto, essa ainda é uma realidade de grande parte dos adolescentes brasileiros. Dados na literatura sobre adesão e retenção de participantes, em modelos de programas de saúde conduzidos em contexto internacional, variam entre 20-30\%, podendo chegar a 50\% de perda ao longo da intervenção (Riekert, Ockene, \& Pbert, 2014). Dessa forma, embora tenha havido desistência de participantes neste estudo, foi possível a avaliação da proposta de intervenção. Estudos futuros com um número maior de adolescentes, em delineamento quase-experimental, possibilitarão análises intra e entre grupos, garantindo maior poder dos testes estatísticos.

A avaliação da intervenção pelos participantes ocorreu no último encontro. Em relação à Satisfação com a intervenção, as pontuações mais baixas foram observadas nos itens tempo e horário dos encontros. Registros do Diário de campo demonstram que as participantes manifestaram o interesse em permanecer mais tempo nos encontros e na escola. Portanto, a escola pode valorizar esse interesse dos seus alunos, promovendo atividades que incentivem engajamento efetivo entre seus estudantes como uma forma não só de melhorar o rendimento escolar, mas também de estabelecer vínculos e amizades duradouras (Silva, Veiga, Pinto, \& Ribas, 2017). Quanto ao horário, muitas das participantes chegavam aos encontros com atraso. Os encontros aconteceram no turno inverso de aulas, com um intervalo de duas horas entre o final da aula da manhã e o início das atividades à tarde, o que pode ter comprometido a pontualidade, pois muitas delas ainda 
organizavam as tarefas de casa antes de irem para a escola. Apesar do horário estabelecido, optou-se por aguardar entre 10 e 15 minutos para iniciar os encontros, de forma a não prejudicar quem chegasse mais tarde. Acredita-se que, quando se desenvolve uma intervenção em turno inverso, o pesquisador deve ser flexível em relação aos horários, pois seu interesse é manter a adesão dos participantes, compreendendo a sua realidade. Para Günther (2006), são características da pesquisa qualitativa sua grande flexibilidade e adaptabilidade. É importante que o contexto onde o estudo está sendo conduzido seja considerado nos procedimentos de pesquisa.

Analisando os resultados do item da Medida de Avaliação da Intervenção, quanto à Clareza e compreensão dos conteúdos, o item com pontuação mais baixa refere-se ao entendimento dos temas abordados. Ressalta-se que todos os temas estão inter-relacionados; portanto, as ausências dos participantes em alguns encontros podem justificar esse escore, ainda que, a cada novo encontro, a moderadora realizasse uma retrospectiva do que foi abordado anteriormente. Assim sendo, para novas replicações da intervenção, deve ser estipulado um número mínimo de participação nos encontros, com vistas a não prejudicar a compreensão dos participantes quanto aos temas de cada encontro.

No item Aplicação dos conteúdos na vida diária, a questão com média mais baixa foi "Quanto você aplicou na sua vida cotidiana os conteúdos abordados na intervenção?”. Esse item, diferentemente dos demais, refere-se a uma percepção mais subjetiva, que necessita de reflexão acerca das próprias experiências e de uma análise contextual da escola. Nesse sentido, é possível supor que as adolescentes ainda não estejam valorizando as suas próprias contribuições ou que seja necessário mais tempo após o término da intervenção para uma análise das mudanças no ambiente escolar, a partir de suas próprias percepções.

A análise das Habilidades sociais e da Integridade/Fidelidade do moderador é um critério metodológico utilizado para avaliar a eficácia de uma intervenção, e esse processo é realizado por um observador em cada encontro, que utiliza um instrumento de avaliação (Gottfredson et al., 2015). Com essas anotações, a moderadora tem a possibilidade de rever sua performance de forma a cumprir todos os critérios estabelecidos e aperfeiçoar aspectos que não estejam sendo atendidos. Essa avaliação de cada encontro é tão relevante quanto a implementação da intervenção e deve ser parte integrante do processo de desenvolvimento de uma intervenção.

No processo de análise qualitativa dos dados, o tema "Aplicação das aprendizagens" pode ser relacionado ao item Aplicação do conteúdo na vida diária constante na Medida de Avaliação da Intervenção. Ainda que na avaliação quantitativa esse item tenha apresentado média mais baixa, percebeu-se que as adolescentes compreenderam conceitos, como empatia e respeito, tendo aplicado essa aprendizagem ao auxiliarem colegas em momentos difíceis ou tristes. Na adolescência, as relações entre pares tornam-se mais significativas, e essas experiências de amizade e companheirismo no ambiente escolar, também promovidas durante a intervenção, podem ser consideradas mecanismos de proteção (Harmelen et al., 2017). Assim, a escola tem o papel de favorecer relações positivas entre os estudantes, de forma que se constitua em um espaço protetivo para prevenir e mediar situações de violência escolar.

$\mathrm{Na}$ análise do segundo tema, "Engajamento e protagonismo", foi observado que as participantes se sentiram parte integrante da escola e protagonistas no processo de prevenção do bullying. Conduziram atividades com o grupo de crianças conforme planejado, ainda que, em alguns momentos, tenham demonstrado timidez ao se expor, possivelmente por não ser algo realizado com frequência por elas. O engajamento reduz as probabilidades de envolvimento em bullying (Forster et al., 2019), assim sendo, é válido que a escola proponha ações extraclasse, capazes de promover o envolvimento efetivo de estudantes na escola (Lewis et al., 2011). A proposta desta intervenção é possibilitar aos estudantes que compreendam as dinâmicas do bullying e seus temas inter-relacionados, mas também que se tornem multiplicadores junto a outros colegas. Os participantes têm o potencial de atuarem como protagonistas na prevenção do bullying junto a seus pares, pois conhecem melhor os problemas que lhes afetam diretamente (Tognetta et al., 2019). 
No tema "Avaliação da intervenção", relatos das adolescentes e anotações do Diário de Campo permitiram concluir que a avaliação foi positiva. A opção por atividades que promovam o engajamento e o protagonismo pode ter sido um fator importante nessa avaliação. A estratégia de valorizar os conhecimentos dos participantes e torná-los agentes de prevenção ao bullying é utilizada em intervenções já avaliadas positivamente, como o OBPP (Olweus \& Limber, 2010).

\section{Considerações Finais}

Programas de intervenção antibullying têm sido desenvolvidos sob diferentes perspectivas, na tentativa de reduzir e minimizar os danos causados por essa forma de violência no contexto escolar. A partir do pressuposto de que escolas brasileiras devem implementar medidas de conscientização e prevenção ao bullying, conforme a lei 13.185/15, foi delineada a intervenção \#NoBullying, destinada a adolescentes escolares. Neste primeiro momento, foi realizado, então, este estudo de viabilidade.

Quanto às limitações do estudo, é necessário considerar que a intervenção é direcionada apenas aos alunos, sem envolver toda a comunidade escolar, como professores e funcionários, gestores e famílias. Além disso, a amostra foi reduzida e somente contou com a adesão de meninas. Em estudos futuros, será necessário considerar esses aspectos, buscando uma maior diversificação do grupo de participantes e o envolvimento de toda a comunidade escolar. Apesar das limitações, concluiu-se que os resultados foram satisfatórios e importantes para a condução futura do estudo quase-experimental de grupos, com avaliação pré-teste e pós-teste nos grupos experimental e controle, realizando-se todas as alterações necessárias identificadas neste estudo de viabilidade. A partir da avaliação da intervenção, considera-se que a proposta tem potencial para contribuir na prevenção ao bullying, promover um papel mais ativo dos estudantes e relações mais positivas no ambiente escolar, e pode ser replicada com outros grupos de adolescentes.

O bullying escolar é um problema de saúde pública que deve ser prevenido com medidas eficazes e condizentes com a realidade de cada instituição de ensino. Dessa forma, é importante que instituições escolares e gestores públicos reconheçam as consequências negativas do bullying e a necessidade de preveni-lo, solidificando-se como agentes de proteção de estudantes vitimizados diretamente ou indiretamente por essa forma de violência. Assim, combater o bullying significa cumprir com a legislação vigente e contribuir para a transformação da realidade social dos estudantes, para que reconheçam a escola como um espaço adequado para criar novos vínculos, adquirir novos conhecimentos e fortalecer-se enquanto grupo.

\section{Referências}

Albores-Gallo, L., Sauceda-García, J. M., Ruiz-Velasco, S., \& Roque-Santiago, E. (2011). El acoso escolar (bullying) y su asociación con trastornos psiquiátricos en una muestra de escolares en México. Salud Pública de México, 53(3), 220-227. http://www.scielo.org.mx/pdf/spm/v53n3/a06v53n3.pdf

American Psychological Association (APA, 2002). Criteria for evaluating treatment guidelines. American Psychologist, 57, $1052-1059$.

Arango, A., Opperman, K. J., Gipson, P. Y., \& Kin, C. A. (2016). Suicidal ideation and suicide attempts among youth who report bully victimization, bully perpetration and/or low social connectedness. Journal of Adolescence, 51, 19-29. https://doi.org/10.1016/j.adolescence.2016.05.003

Bonell, C., Allen, E., Warren, E., McGowan, J., Bevilacqua, L., Jamal, F., Legood, R., Wiggins, M., Opondo, C., Mathiot, A., Sturgess, J., Fletcher, A., Sadique, Z., Elbourne, D., Christie, D., Bond, L., Scott, \& S., Viner, R. M. (2018). Effects of the Learning Together Intervention on bullying and aggression in English secondary schools (INCLUSIVE): a cluster randomised controlled trial. The Lancet, 392(10163), 2452-2464. https://doi.org/10.1016/S01406736(18)31782-3

Brito, R., \& Koller, Silvia H. (1999). Desenvolvimento humano e redes de apoio social e afetivo. In A. M. Carvalho (Ed.), O mundo social da criança: natureza e cultura em ação (pp. 115-126). Casa do Psicólogo.

Calvete, E., Orue, I., Fernández-González, L., \& Prieto-Fidalgo, A. (2019). Effects of an incremental theory of personality intervention on the reciprocity between bullying and cyberbullying victimization and perpetration in adolescents. PLOS ONE, $14(11)$, e0224755. https://doi.org/10.1371/journal.pone.0224755

Cosma, A., Walsh, S. D., Chester, K. L., Callaghan, M., Molcho, M., Craig, W., \& Pickett, W. (2020). Bullying victimization: time trends and the overlap between traditional and cyberbullying across countries in Europe and North America. International Journal of Public Health, 65, 75-85. https://doi.org/10.1007/s00038-019-01320-2 
Costa, A. C. G. (2000). Protagonismo juvenil: adolescência, educação e participação democrática. undação Odebrecht, 2000.

Creswell, J. W. (2003). Research design: qualitative, quantitative, and mixed methods approaches. Sage. (Originalmente publicado em 1994).

Durgante, H. B. (2019). Desenvolvimento, implementação e avaliação do programa Vem Ser: programa de psicologia positiva para a promoção de saúde de aposentados. Tese de Doutorado, Programa de Pós-Graduação em Psicologia, Universidade Federal do Rio Grande do Sul, Porto Alegre, RS.

Ferreira, T. R. Z. C., \& Deslandes S. F. (2018). Cyberbullying: conceituações, dinâmicas, personagens e implicações à saúde. Ciência \& Saúde Coletiva, 23(10), 3369-3379. https://doi.org/10.1590/1413-812320182310.13482018

Fleming, L. C., \& Jacobsen, K. H. (2010). Bullying among middle-school students in low and middle income countries. Health Promotion International, 25(1), 73-84 https://doi.org/10.1093/heapro/dap046

Forster, M., Gower, A. L., Gloppen, K., Sieving, R., Oliphant, J., Plowman, S., Gadea, A., \& MacMorris, B. (2019). Associations between dimensions of school engagement and bullying victimization and perpetration among middle school students. School Mental Health. Advance online publication. https://doi.org/10.1007/s12310-019-09350-0

Fredricks, J. A., Blumenfeld, P. C., \& Paris, A. H. (2004). School engagement: potential of the concept, state of the evidence. Review of Educational Research Spring, 74(1), 59-109. https://doi.org/10.3102/00346543074001059

Gottfredson, D. C., Cook, T. D., Gardner, F. E., Gorman-Smith, D., Howe, G. W., Sandler, I. N., \& Zafft, K. M. (2015). Standards of evidence for efficacy, effectiveness, and scale-up research in prevention science: next generation. Prevention Science, 16(7), 893-926. https://doi.org/10.1007/s11121-015-0555-X

Günther, H. (2006). Pesquisa qualitativa versus pesquisa quantitativa: esta é a questão? Psicologia: Teoria $e$ Pesquisa, 22(2), 201-209. https://doi.org/10.1590/S0102-37722006000200010

Harmelen, A. L., Kievit, R. A., Ioannidis, K., Neufeld, S., Jones, P. B., Bullmore, E., Dolan, R., The NSPM Consortium, Fonagy, P., \& Goodyer, I. (2017). Adolescent friendships predict later resilient functioning across psychosocial domains in a healthy community cohort. Psychological Medicine, 47, $2312-2322$. https://doi.org/10.1017/S0033291717000836

Instituto Brasileiro de Geografia e Estatística (IBGE, 2015). Trabalho infantil e trabalho infantil doméstico no Brasil: Avaliação a partir dos microdados da Pnad/IBGE (2012-2013). Rio de Janeiro: IBGE.

Juvonen, J., Schacter, H. L., Sainio, M., \& Salmivalli, C. (2016). Can a school-wide bullying prevention program improve the plight of victims? Evidence for risk intervention effects. Journal of Consulting and Clinical Psychology, 84(4), 334-344. https://doi.org/10.1037/ccp0000078

Lei N. 13.185, de 06 de novembro de 2015. (2015). Institui o Programa de Combate à Intimidação Sistemática (Bullying). Recuperado de http://www.planalto.gov.br/ccivil_03/_ato2015-2018/2015/lei/113185.htm

Lewis, A. D., Huebner, S., Malone, P. S., \& Valois, R. F. (2011). Life satisfaction and student engagement in adolescents. Journal Youth Adolescence, 40, 249-262. https://doi.org/10.1007/s10964-010-9517-6

Li, J., Sidibe, A. M., Shen, X., \& Hesketh, T. (2019). Incidence, risk factors and psychosomatic symptoms for traditional bullying and cyberbullying in Chinese adolescents. Children and Youth Services Review, 107, 1-8. https://doi.org/10.1016/j.childyouth.2019.104511

Lopes, A. L. M., Silva, S. A., Castro, D. F. A., Bógus, C. M., \& Fracolli, L. A. (2013). Avaliação de programas, serviços e tecnologias na perspectiva da promoção da saúde: uma reflexão teórica. Revista Brasileira em Promoção da Saúde, 26(4), 590-594. http://dx.doi.org/10.5020/18061230.2013.p590

Lopez, S. J. (Ed.). (2009). The encyclopedia of positive psychology. Wiley-Blackwell.

Malta, D. C., Porto, D. L., Crespo, C. D., Silva, M. M. A., Andrade, S. S. C., Mello, F. C. M., Monteiro, R., \& Silva, M. A. I. (2014). Bullying em escolares brasileiros: análise da Pesquisa Nacional de Saúde do Escolar (PeNSE 2012). Revista Brasileira de Epidemiologia, 17(1), 92-105. https://doi.org/10.1590/1809-4503201400050008.

Martín-Quintana, J. C., Chaves, M. L. M., López, M. J. R., Byme, S., Ruiz, B. R., \& Suárez, G. R. (2009). Programas de Educación Parental. Intervención Psicosocial, 18(2), 121-133. https://scielo.isciii.es/pdf/inter/v18n2/v18n2a04.pdf

Mello, F. C. M., Silva, J. L., Oliveira, W. A., Prado, R. R., Malta, D. C., \& Silva, M. A. I. (2017). A prática de bullying entre escolares brasileiros e fatores associados, Pesquisa Nacional de Saúde do Escolar 2015. Ciência \& Saúde Coletiva, 22(9), 2939-2948. https://doi.org/10.1590/1413-81232017229.12762017

Menard, S., \& Grotpeter, J. K. (2014). Evaluation of bully-proofing your school as an elementary school antibullying intervention. Journal of School Violence, 13, 188-209. https://doi.org/10.1080/15388220.2013.840641

National Institute for Health Research (NIHR, 2017). Feasibility and pilot studies: which programme should I apply to? http://www.nets.nihr.ac.uk/glossary

Olweus, D. (1993). Bullying at school. Blackwell Publishing.

Olweus, D., \& Limber, S. P. (2010). Bullying in school: evaluation and dissemination of the Olweus Bullying Prevention Program. American Journal of Orthopsychiatry, 80(1), 124-134. https://doi.org/10.1111/j.1939-0025.2010.01015.x

Organização das Nações Unidas (ONU). (2014). Relatório mundial sobre a prevenção da violência 2014. https://nev.prp.usp.br/wpcontent/uploads/2015/11/1579-VIP-Main-report-Pt-Br-26-10-2015.pdf

Palladino, B. E., Nocentini, A., \& Menesini, E. (2016). Evidence-based intervention against bullying and cyberbullying: Evaluation of the NoTrap! program in two independent trials. Aggressive Behavior, 42(2), 194-206. https://doi.org/10.1002/ab.21636 
Rana, M., Gupta, M., Malhi, P., Grover, S., \& Kaur, M. (2018). Effectiveness of a multicomponent school based intervention to reduce bullying among adolescents in Chandigarh, North India: A quasi-experimental study protocol. Journal of Public Health Research, 7(1304), 50-55. https://doi.org/10.4081/jphr.2018.1304

Riekert, K. A., Ockene, J. K., \& Pbert, L. (Eds.). (2014). The handbook of health behavior change. Spring.

Saldaña, J. (2009). The coding manual for qualitative researchers. Sage.

Salmivalli, C. (2010). Bullying and the peer group: a review. Aggression and Violent Behavior, 15(2), 112-120. https://doi.org/10.1016/j.avb.2009.08.007

Santana, J. P., \& Avanzo, J. R. (2014). Infância e direitos: o uso de metodologias participativas em contexto de acolhimento institucional. Revista Subjetividades, 14(2), 306-318. http://pepsic.bvsalud.org/pdf/rs/v14n2/13.pdf

Seligman, M. E. P. (2002). Authentic happiness: Using the new Positive Psychology to realize your potential for lasting fulfillment. London: Nicholas Brealey Publishing.

Seligman, M. E. P., Steen, T. A., Park, N., \& Peterson, C. (2005). Positive Psychology progress: empirical validation of interventions. American Psychologist, 60, 410-421. https://doi.org/10.1037/0003-066x.60.5.410

Silva, J. L., Oliveira, W. A., Braga, I. F., Farias, M. S., Lizzi, E. A. S., Gonçalves, M. F. C., Pereira, B. O., \& Silva, M. A. I. (2016). The effects of a skillbased intervention for victims of bullying in Brazil. International Journal Environmental Research Public Health, 13(1042), 2-10. https://doi.org/10.3390/ijerph13111042

Silva, C. R., Veiga, F., Pinto, E. S., \& Ribas, A. (2017). Envolvimento dos alunos na escola e suas perceções acerca do apoio parental. Revista de Estudios e Investigación en Psicología y Educación, extra(5), 28-32. https://doi.org/10.17979/reipe.2017.0.05.2189

Silva, J. L., Oliveira, W. A., Carlos, D. M., Lizzi, E. A. S., Rosário, R., \& Silva, M. A. I. (2018a). Intervenção em habilidades sociais e bullying. Revista Brasileira de Enfermagem, 71(3), 1150-1156. https://doi.org/10.1590/0034-7167-2017-0151

Silva, J. L., Oliveira, W. A., Zequinão, M. A., Lizzi, E. A. S., Pereira, B. O., \& Silva, M. A. I. (2018b). Resultados de intervenções em habilidades sociais na redução de bullying escolar: revisão sistemática com metanálise. Temas em Psicologia, 26(1), 509-522. https://doi.org/10.9788/TP2018.1-20Pt

Smith, P. K. (2011). Why interventions to reduce bullying and violence in schools may (or may not) succeed: Comments on this Special Section. International Journal of Behavioral Development, 35(5), 419-423. https://doi.org/10.1177/0165025411407459

Smith, P. K. (2014). Understanding school bullying: its nature \& prevention strategies. Sage.

Smith, P. K., Görzig, A., \& Robinson, S. (2018). Issues of cross-cultural variation in cyberbullying across Europe and beyond. Media@LSE Working Paper Series, 49, 1-28. https://repository.uwl.ac.uk/id/eprint/4786/

Smith, P. K., Sundaram, S., Sandhu, D., Blaya, C., Schäfer, M., \& Spears, B. (2018). Introduction. In Smith, P. K., Sundaram, S., Sandhu, D., Blaya, C., Schäfer, M., \& Spears, B. Bullying, cyberbullying and student well-being in schools: Comparing European, Australian and Indian perspectives. London: Cambridge University. https://doi.org/10.1017/9781316987384

Tickle-Degnen, L. (2013). Nuts and bolts of conducting feasibility studies. American Journal of Occupational Therapy, 67(2), 171-176. https://doi.org/10.5014/ajot.2013.006270

Tognetta, L. R. P., Souza, R. A., \& Lapa, L. Z. (2019). A implantação das equipes de ajuda como estratégia para a superação do bullying escolar. Revista Educação PUC-Campinas, 24(3), 397-410. https://doi.org/10.24220/2318-0870v24n3a4506

Vivaldi, F. M. C. (2020). A função social da escola: a implantação de um projeto institucional para a convivência ética. Tese de Doutorado, Programa de PósGraduação em Educação, Faculdade de Educação, Universidade Estadual de Campinas, Campinas, 2010. 